\title{
Plasticidade alimentar em Rivulus pictus Costa (Osteichthtyes, Cyprinodontiformes, Rivulidae) de uma pequena lagoa em Brasília, Distrito Federal, Brasil
}

\author{
Oscar Akio Shibatta \& Sirlei Terezinha Bennemann
}

Departamento de Biologia Animal e Vegetal, Centro de Ciências Biológicas, Universidade Estadual de Londrina. 86051-990 Londrina, Paraná, Brasil.

\begin{abstract}
Feeding plasticity of Rivulus pictus Costa (Osteichthyes, Cyprinodontiformes, Rivulidae) from a small lake in Brasília, Distrito Federal, Brazil. Rivulus pictus has a wide distribution in Distrito Federal and lives in different environments, including small lakes. The analysis of gastric and intestinal contents showed that most of the 21 ingested food items were from autochthonous origin and characterized the diet of an omnivorous fish with a trend to planktivory. Five feeding items were important in the composition of the alimentary spectrum and revealed that food resources are explored near water surface (Acari), in the water column (Copepoda, Cladocera and Closterium sp.) and also in the bottom (Chironomidae). The varied diet and the capacity to explore all levels of the environment evidenced that feeding flexibility is one of the factors that facilitates the survival of this species in different environments.
\end{abstract}

KEY WORDS. Diet, feeding spectrum, ecology.

\begin{abstract}
A biologia e a ecologia de peixes de ambientes como pequenas lagoas e brejos são pouco estudadas no Brasil. Tais corpos de água constituem-se em ambientes particulares, com importância ecológica nos ecossistemas em que se encontram, visto que muitos animais os utilizam como fonte de água. As alterações antrópicas, tais como drenagens e aterramentos (e.g. Costa 2002), podem acarretar a extinção de populações de peixes e outros organismos que desenvolvem todo o seu ciclo de vida nesses habitats.

Um dos fatores que podem estar relacionados à sobrevivência de peixes nestes ambientes é a plasticidade alimentar. Um amplo espectro alimentar é adequado e muito comum nos peixes de água doce fluviais quando mudanças nas condições ambientais acarretam alterações na disponibilidade de alimentos (Lowe-McConnell 1999, Abelha et al. 2001).

O guaru Rivulus pictus Costa, 1989 vive em pequenas lagoas, brejos e riachos no Distrito Federal, mas o pequeno porte e o seu exíguo valor comercial talvez sejam fatores que pouco estimularam os pesquisadores para o seu estudo. Entretanto, é uma espécie ecologicamente importante, pois a capacidade de se dispersar movendo-se fora da água, quando as condições ambientais se tornam adversas, possibilita colonizar e atuar em diferentes ambientes aquáticos. A sua ampla distribuição é facilitada pela capacidade de sobreviver até $60 \mathrm{~h}$ fora da água, conforme foi verificado por E.K. Bastos (dados não publicados).
\end{abstract}

Este é o primeiro estudo que investiga a dieta da espécie $R$. pictus e sua variação ao longo de dez meses, numa pequena lagoa. Desta forma, pretende-se compreender os fatores relacionados ao seu sucesso de sobrevivência, pelo conhecimento da composição da dieta natural. Além disso, é de interesse investigar se a dieta inclui larvas de mosquitos hematófagos, cuja ocorrência nessas águas poderia ser aventada.

\section{MATERIAL E MÉTODOS}

A espécie foi identificada segundo Costa (1989) e o material testemunho está depositado no Museu de Zoologia da Universidade Estadual de Londrina, Paraná. As amostragens de $R$. pictus foram feitas mensalmente numa pequena lagoa de aproximadamente $10 \mathrm{~m}$ de diâmetro, profundidade de 20 a 40 $\mathrm{cm}$, com substrato lodoso, transparência da água total, no final da Asa Norte de Brasília, Distrito Federal, localizada a $15^{\circ} 43^{\prime} 57,3^{\prime \prime}$ S e $47^{\circ} 53^{\prime} 27,3^{\prime \prime} \mathrm{W}$. Provavelmente esta lagoa é o resquício de uma lagoa natural, que permaneceu no local, mesmo após a implantação das avenidas asfaltadas. A forma da lagoa, bem como as características de suas proximidades são mostradas na figura 1 . Os exemplares foram capturados com peneira nos meses de março a dezembro de 1989, no período de 9:00 às 10:00 h, fixados em formol 10\% e preservados em álcool $70 \%$. O comprimento padrão foi medido com paquímetro com precisão de $0,05 \mathrm{~mm}$. 


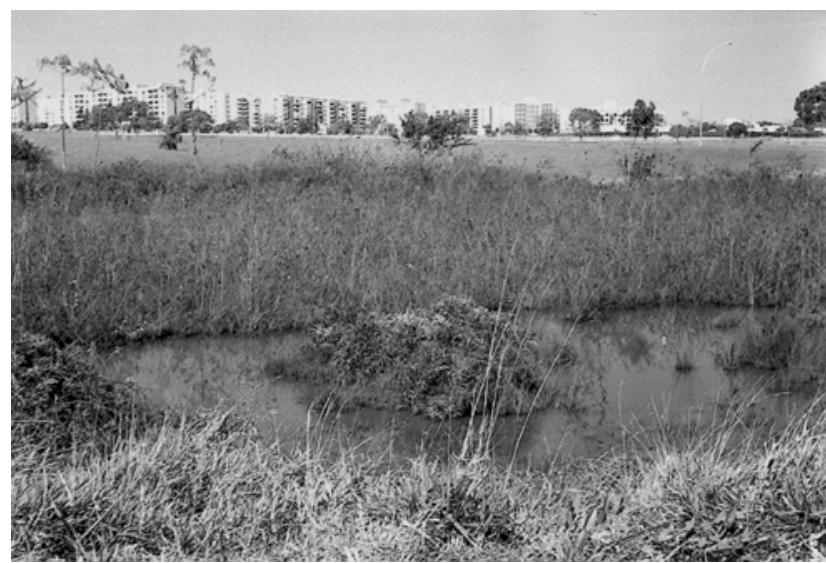

Figura 1. Vista geral da área de coleta de Rivulus pictus em Brasília, março de 1989 (foto de Hélio M. Shibatta).

O conteúdo gastro-intestinal de 10 exemplares foi analisado por mês, com lupa estereoscópica e os organismos encontrados identificados até o nível taxonômico mais restrito possível. Todos os alimentos consumidos foram analisados pelos métodos de freqüência de ocorrência e numérico (Hyslop 1980). Massas de algas filamentosas ou de detritos foram contabilizados como uma unidade por exemplar. Os itens alimentares foram somados para o cálculo de seus percentuais mensais e total. A relação entre as porcentagens da freqüência de ocorrência e abundância foi utilizada para a análise gráfica, segundo Costello (1990). Os itens alimentares, com valores maiores que as médias das freqüências de ocorrência totais e das percentagens numéricas totais, foram considerados como importantes para a espécie.

\section{RESULTADOS E DISCUSSÃO}

Foram analisados 100 exemplares no total, cujo comprimento padrão $(\mathrm{CP})$ variou de 12 a $25,2 \mathrm{~mm}$ (média 18,2 $\mathrm{mm}$, $\mathrm{n}=99$ ). O sistema gastro-intestinal tem a forma de " $\mathrm{Z}$ " e é composto por um estômago grande e um intestino curto.

Apesar da dieta ser composta por 21 itens alimentares, 14 foi o número máximo consumido em cada amostra. Os principais itens, em percentuais totais de abundância e de freqüência de ocorrência dos meses analisados foram Chironomidae, Cladocera e Closterium sp., registrados em todas as amostras analisadas, além de Acari, Copepoda e Diptera adulto que figuraram em 90\% dos meses (Tab. I). Através dos percentuais totais é possível listar os alimentos na seguinte ordem decrescente de importância: Cladocera, Copepoda, Closterium sp., Chironomidae e Acari. A abundância numérica indicou que estes cinco itens principais representaram $83,4 \%$ da quantidade total de organismos ingeridos (Tab. I), o que sugere uma forte tendência à planctivoria.

Os recursos alimentares principais utilizados por R. pictus, no ambiente de lagoa, destacaram-se por ser quase na sua totalidade autóctones quanto à fonte de origem. Taylor (1992), estudando Rivulus marmoratus Poey, 1880 da Flórida, verificou que, apesar da espécie ter ingerido alimentos de origem alóctone, os maiores números e as maiores freqüências de itens

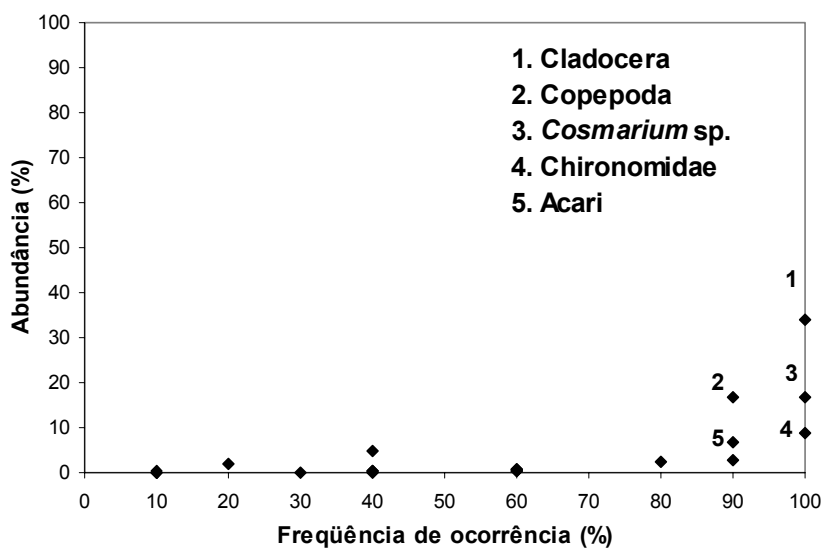

Figura 2. Representação gráfica das percentagens das freqüências de ocorrência e das abundâncias dos itens alimentares consumidos por Rivulus pictus.

alimentares foram os de origem autóctone. Shibatta \& Rocha (2001) verificaram que o pirá-brasília, Simpsonichthys boitonei Carvalho, 1959 de um brejo da Reserva Ecológica do Instituto Brasileiro de Geografia e Estatística, no Distrito Federal, também se alimentou principalmente de itens de origem autóctone, mostrando que as espécies de peixes que habitam estes pequenos ambientes tem um aproveitamento dos organismos que também desenvolvem todo o seu ciclo de vida no local.

Esse amplo espectro alimentar é favorecido pelo ambiente lêntico, como é caracterizada a lagoa. Em ambiente lótico os alimentos disponíveis na coluna da água são menos abundantes como, por exemplo, os microcrustáceos (Luiz et al. 1998) e é verificada predominância de dietas de origem alóctone em peixes de riachos (Lowe-McConnell 1999, Esteves \& Aranha 1999). Mesmo assim, é possível deduzir que R. pictus é capaz de sobreviver em riachos por explorar recursos alimentares de diferentes extratos da coluna da água, inclusive os do fundo.

Os cinco tipos de recursos que se destacaram na composição da dieta de $R$. pictus, revelam como esses são explorados desde próximo à superfície (Acari), na coluna da água (Copepoda, Cladocera e Closterium sp.) e no fundo (Chironomidae).

Apesar da tendência da análise numérica em valorizar os pequenos itens alimentares que ocorrem em grande quantidade, e em todo o tempo, nota-se também a importância dos itens de grande tamanho, como foi o caso de Odonata, cujas náiades foram abundantes em poucos meses (outubro e novembro) apesar de ser constante, na grande maioria dos meses analisados (Tab. I).

Quanto à importância desta espécie de peixe no combate aos mosquitos hematófagos, não foi verificada a presença de larvas na dieta. No entanto, devido ao amplo espectro alimentar, não se deve descartar a hipótese de que eles possam ser possíveis controladores da população destes insetos.

$\mathrm{Na}$ preocupação constante para erradicar focos de criação de mosquitos hematófagos, muitas vezes transmissores de moléstias que acometem a espécie humana, têm-se utilizado espécies exóticas que nem sempre constituem a melhor solução para o problema (Elton 2000) como pode ser verificado no uso do famoso barrigudinho (Poecilia reticulata Peters, 1859) 
Tabela I. Número total, percentual de abundância (PN\%) e freqüência de ocorrência (FO\%) de organismos encontrados mensalmente nos conteúdos estomacais de Rivulus pictus (10 exemplares examinados por mês).

\begin{tabular}{|c|c|c|c|c|c|c|c|c|c|c|c|c|c|}
\hline Item alimentar & Mar & Abr & Mai & Jun & Jul & Ago & Set & Out & Nov & Dez & Total & PN\% & FO\% \\
\hline Acari & 37 & 6 & 0 & 2 & 3 & 3 & 4 & 3 & 7 & 38 & 103 & 6,61 & 90 \\
\hline Algas filamentosas & 0 & 2 & 0 & 1 & 1 & 2 & 1 & 1 & 1 & 0 & 9 & 0,58 & 60 \\
\hline Aranha & 0 & 0 & 0 & 0 & 4 & 1 & 3 & 0 & 2 & 1 & 11 & 0,70 & 60 \\
\hline Arthropoda (restos) & 0 & 3 & 1 & 0 & 0 & 0 & 4 & 0 & 1 & 0 & 9 & 0,58 & 40 \\
\hline Chironomidae & 22 & 10 & 15 & 7 & 11 & 11 & 11 & 20 & 24 & 7 & 138 & 8,85 & 100 \\
\hline Cladocera & 35 & 101 & 113 & 24 & 64 & 49 & 55 & 53 & 28 & 8 & 530 & 33,97 & 100 \\
\hline Closterium sp. & 115 & 62 & 18 & 4 & 1 & 5 & 10 & 16 & 23 & 9 & 263 & 16,86 & 100 \\
\hline Copepoda & 0 & 10 & 45 & 55 & 9 & 62 & 56 & 5 & 7 & 18 & 267 & 17,12 & 90 \\
\hline Cosmarium sp. & 0 & 0 & 0 & 0 & 0 & 0 & 0 & 9 & 0 & 0 & 9 & 0,58 & 10 \\
\hline Detritos & 1 & 2 & 2 & 3 & 0 & 0 & 0 & 2 & 4 & 0 & 14 & 0,90 & 60 \\
\hline Diptera (adulto) & 5 & 2 & 3 & 8 & 4 & 8 & 5 & 5 & 0 & 4 & 44 & 2,82 & 90 \\
\hline Diptera (ovos, larvas e pupas) & 0 & 2 & 0 & 0 & 0 & 0 & 7 & 0 & 4 & 52 & 65 & 4,17 & 50 \\
\hline Euastrum sp. & 0 & 0 & 0 & 0 & 0 & 0 & 0 & 1 & 0 & 0 & 1 & 0,06 & 10 \\
\hline Hemiptera & 1 & 0 & 0 & 0 & 1 & 0 & 1 & 1 & 3 & 2 & 9 & 0,58 & 60 \\
\hline Hymenoptera & 0 & 1 & 0 & 0 & 0 & 0 & 1 & 0 & 0 & 1 & 3 & 0,19 & 20 \\
\hline Insecta & 0 & 0 & 1 & 0 & 4 & 1 & 0 & 0 & 2 & 0 & 8 & 0,51 & 40 \\
\hline Odonata & 2 & 1 & 1 & 0 & 0 & 1 & 1 & 12 & 18 & 4 & 40 & 2,56 & 80 \\
\hline Ostracoda & 0 & 0 & 0 & 0 & 0 & 0 & 21 & 0 & 0 & 9 & 30 & 1,92 & 20 \\
\hline Sedimento & 0 & 0 & 0 & 0 & 0 & 0 & 0 & 0 & 1 & 0 & 1 & 0,06 & 10 \\
\hline Tabanidae (larvas) & 1 & 1 & 0 & 0 & 0 & 0 & 0 & 0 & 0 & 1 & 3 & 0,19 & 30 \\
\hline Trichoptera & 3 & 0 & 0 & 0 & 0 & 0 & 0 & 0 & 0 & 0 & 3 & 0,19 & 10 \\
\hline Total & 222 & 203 & 199 & 104 & 102 & 143 & 180 & 128 & 125 & 154 & 1560 & 100,00 & \\
\hline
\end{tabular}

das ilhas de Trinidad e norte da América do Sul. Provavelmente esta espécie foi a mais difundida não apenas nos trópicos, mas também em países de clima temperado, como a Alemanha e Estados Unidos, onde atuou como forte competidora com as espécies nativas (Coates \& Atz 1964). Desta maneira, para evitar um comprometimento grave no ecossistema aquático, há a necessidade de se conhecer quais as espécies nativas que mais se adaptam à função de controladores biológicos.

Na figura 2 a importância dos cinco principais itens alimentares se torna mais evidente, pois os números foram superiores às médias da percentagem numérica $(4,76 \%)$ e da freqüência de ocorrência $(53,8 \%)$. Numa síntese, é possível inferir que Rivulus pictus é uma espécie generalista, pois utiliza um grande número de recursos alimentares, mas aproveita os de maior disponibilidade, como era de se esperar para uma espécie que sobrevive em diferentes tipos de ambientes.

\section{AGRADECIMENTOS}

A Antônio José Andrade Rocha pelas orientações iniciais do trabalho. A Hélio Minoru Shibatta pelo auxílio em campo, pelas informações das coordenadas geográficas e foto do local de coletas. A Cláudia Bueno dos Reis Martinez pelas correções do abstract. A Lenice de Souza e aos assessores da RBZ pelas valiosas sugestões ao artigo. Ao CNPq pela bolsa de Iniciação Científica concedida ao autor sênior (proc. 801746/88-4).

\section{REFERÊNCIAS BIBLIOGRÁFICAS}

Abelha, M.C.F.; A.A. Agostinho \& E. Goulart. 2001. Plasticidade trófica em peixes de água doce. Acta Scientiarum, Maringá, 23: 425-434.

Coats, C.W. \& J.W. Atz. 1964 The illustrated encyclopedia of animal life, vol. 12. Londres: Odhams Book Ltd., pp. 13931534.

Costa, W.J.E.M. 1989. Descrição de cinco novas espécies de Rivulus das bacias dos rios Paraná e São Francisco (Cyprinodontiformes, Rivulidae). Revista Brasileira de Zoologia, Curitiba, 6 (3): 523-534.

. 2002. Peixes anuais brasileiros. Diversidade e conservação. Curitiba, Editora Universidade Federal do Paraná, 238p.

Revista Brasileira de Zoologia 20 (4): 615-618, dezembro 2003 
Costello, M.J. 1990. Predator feeding strategy and prey importance: a new graphical analysis. Journal of Fish Biology, London, 36: 261-263.

Elton, C.S. 2000. The ecology of invasions by animal and plants. Chicago, The University of Chicago Press, XIV+181.

Esteves, K.E. \& J.M.R. Aranha. 1999. Ecologia trófica de peixes de riachos, p. 157-182. In: E.P. Caramaschi; R. Mazzoni; P.R. Peres-Neto (Eds). Ecologia de peixes de riachos. Rio de Janeiro, PPGE-UFRJ, Série Oecologia Brasiliensis, vol. 6, $\mathrm{XVI}+260 \mathrm{p}$.

Hyslop, E.J. 1980 Stomach contents analysis - a review of methods and their application. Journal of Fish Biology, London, 17: 411-429.

Recebido em 06.III.2003; aceito em 17.X.2003.
Lowe-McConnell, R.H. 1999. Estudos ecológicos de comunidades de peixes tropicais. São Paulo, Edusp, 534p.

Luiz, E.A.; A.A. Agostinho; L.C. Gomes; N.S. Hahn. 1998. Ecologia trófica de peixes em dois riachos da bacia do rio Paraná. Revista Brasileira de Biologia, Rio de Janeiro, 58 (2): 273285.

Shibatta, O.A.; A.J.A. Rocha. 2001. Alimentação em machos e fêmeas do pirá-brasília, Simpsonichthys boitonei Carvalho (Cyprinodontiformes, Rivulidae). Revista Brasileira de Zoologia, Curitiba, 18 (2): 381-385.

Taylor, D.S. 1992. Diet of the killifish Rivulus marmoratus collected from land crab burrows, with further ecological notes. Environmental Biology of Fish, Dordrecht, 33: 389-393. 